\title{
Recombination at Lomer Dislocations in Multicrystalline Silicon for Solar Cells
}

\author{
Jan Bauer, Angelika Hähnel, Peter Werner, Nikolai Zakharov, Horst Blumtritt, Annika Zuschlag, \\ and Otwin Breitenstein
}

\begin{abstract}
Lomer dislocations at small-angle grain boundaries in multicrystalline silicon solar cells have been identified as responsible for the dominating inherent dark current losses. Resulting efficiency losses have been quantified by dark lock-in thermography to be locally up to several percent absolute, reducing the maximum power of the cells. By electron beam induced current measurements and scanning transmission electron microscopy investigations, it is revealed that the strengths of the dark current losses depend on the density of Lomer dislocations at the small-angle grain boundaries.
\end{abstract}

Index Terms-Defects, efficiency losses, Lomer dislocations, mc-Si solar cells, recombination.

\section{INTRODUCTION}

A LTHOUGH using similar production processes and the same silicon feedstock, in general, multicrystalline silicon (mc-Si) solar cells made from vertical gradient freeze (VGF) techniques exhibit lower efficiencies than solar cells made from monocrystalline silicon (mono-Si) [1], [2] made from Czochralski (CZ)-grown Si. These efficiency losses are qualitatively attributed to the higher "material quality" of mono-Si (see, for instance [2]-[5]). What does higher material quality mean? Speaking in terms of the lifetime of minority charge carriers, the lifetime of the carriers in $\mathrm{mc}-\mathrm{Si}$ is locally lower than in mono$\mathrm{Si}$, and therefore, the performance of mc-Si solar cells is lower. The tradeoff between cheaper but reduced-lifetime materials and costly but high-lifetime material was already figured out decades ago (see, for instance [2], [6]). However, it is still one of the major challenges to reveal the inherent material-related loss mechanism in mc-Si solar cells. Although, meanwhile, the growth of the grain structure of multicrystalline VGF Si blocks is controllable [7], and the so-called high performance mc-Si material is available [8], [9], solar cells made from this material

J. Bauer, P. Werner, N. Zakharov, H. Blumtritt, and O. Breitenstein are with the Max Planck Institute of Microstructure Physics, Halle 06120, Germany (email: jbauer@mpi-halle.mpg.de; werner@mpi-halle.mpg.de; zakharov@mpihalle.mpg.de; blumtrit@mpi-halle.mpg.de; breiten@mpi-halle.mpg.de).

A. Hähnel was with the Max Planck Institute of Microstructure Physics, Halle 06120, Germany. She is now with the Fraunhofer IWM, Halle 06120, Germany (e-mail: angelika.haehnel@iwmh.fraunhofer.de).

A. Zuschlag is with the University of Konstanz, Konstanz 78464, Germany (email: annika.zuschlag@uni-konstanz.de). have yet to reach the efficiency of mono-Si cells. In addition, mono-Si solar cells benefit on the process side from textures (e.g., pyramids), which allow better absorption of the incoming light, and therewith, these cells reach higher currents.

During the past couple of years, a strong trend toward Si solar cells with high efficiencies is apparent in research and industry as well [10], [11]. These cells are even stronger dominated by the bulk properties than the first-generation cells. Keeping the costs low is a major issue for all the efforts made. To benefit from the lower production costs of VGF mc-Si in comparison with CZ mono-Si [4], [12], one has to bridge the efficiency gap between the mono-Si and mc-Si material. For this purpose, much more detailed knowledge about the inherent material loss mechanisms in mc-Si is needed.

The main differences between mc-Si and mono-Si materials are the existence of various crystallographic defects in mc-Si like dislocations and various types of grain boundaries (GBs), and the higher contamination with metals, such as $\mathrm{Fe}, \mathrm{Cu}, \mathrm{Ca}$ [13], [14], and nonmetallic impurities like carbon and nitrogen [15] due to the crystallization process. On the other hand, the concentration of oxygen is lower in mc-Si. Metal contaminations in connection with crystallographic defects in silicon are known to affect the minority carrier lifetime (or equivalent: their diffusion length). Hence, in mc-Si solar cells, the so-called saturation current density $J_{01}$, which is strongly dependent on the bulk recombination properties, is significantly higher, compared with mono-Si solar cells, which is one of the major reasons for the inherently lower mc-Si performance. Among the metal impurities, iron is the most prominent being a lifetime killer in $\mathrm{Si}$ (see, for example, [13] and [16]). For mc-Si, this was elaborately investigated for instance by Istratov et al. [13], Buonassisi et al. [14], Coletti et al. [17], [18], and, in combination with special GBs, by Chen et al. [19]. Interstitial iron in $\mathrm{Si}\left(\mathrm{Fe}_{\mathrm{i}}\right)$ is a recombination center according to the Shockley-Read-Hall theory [20]. In boron-doped $\mathrm{Si}$ material, $\mathrm{Fe}_{\mathrm{i}}$ tends to pair with $\mathrm{B}$, leading to iron-boron pairs $(\mathrm{FeB})$, which are less recombination active than $\mathrm{Fe}_{\mathrm{i}}$ [16], [21]. $\mathrm{Fe}_{\mathrm{i}}$ and $\mathrm{FeB}$ are attributed to defect levels with energies of $0.4 \mathrm{eV}$ [22], [23] above the valence band level $E_{\mathrm{V}}$, and $0.26 \mathrm{eV}$ below the conduction band level $E_{\mathrm{C}}[24]-[26]$, respectively.

Indeed, many works about the formation and distribution of $\mathrm{Fe}_{\mathrm{i}}$ and iron precipitates and their impact on the solar cell performance have been published over the past decade. Buonassisi et al., found nanometer-sized iron silicide particles in mc-Si [14], [27], which influence the lifetime. Needle-shaped iron silicide precipitates recently were determined to be the cause of the so-called type- 2 breakdown sites in mc-Si solar cells [28]. 
Schubert et al. [29] showed how the distribution of iron limits the performance of mc-Si solar cells. The sources of iron, its distribution in the forms of $\mathrm{Fe}_{\mathrm{i}}$ and $\mathrm{Fe}$ precipitates, and $\mathrm{Fe}$ gettering effects [30]-[32] during the solar cell manufacturing processes are well investigated. The iron contamination mainly comes from the quartz crucible and the silicon nitride coating of the latter used for the Si ingot production [33]-[36]. There are simulations of the redistribution of $\mathrm{Fe}_{\mathrm{i}}$ and $\mathrm{Fe}$ precipitates during the emitter formation in solar cells [37], [38], as well as results about the distribution and evolution of iron-silicide precipitates in mc-Si [39]. Detailed investigations about the impact of iron concentration and iron distribution in VGF-grown mc-Si ingots on the performance of mc-Si solar cells have been published recently [40], [41].

However, about the detrimental crystallographic defects, only some knowledge exists. In particular, there is a long lasting discussion whether the low lifetime in the poor crystallographic quality regions is caused by more or less randomly distributed dislocations (e.g., [5], [42] and [43]), or by recombination-active GBs (e.g., [44]-[46]). A particular role is played here by smallangle grain boundaries (SAGBs). They are basically rows of dislocations and are by some authors attributed to dislocations and by others to GBs. The recombination behavior of different types of single dislocations in $\mathrm{Si}$ was revealed for instance in [43] and [47], and the impact of the density of dislocations on the recombination activity was shown, e.g., in [48], mostly by electron beam induced current (EBIC) investigations. The results of these investigations were that clean dislocations (i.e., without impurity atoms) are not recombination active at room temperature (RT), only contaminated dislocations may be harmful. The different recombination activities of different types of dislocations have to be attributed to their different gettering activity to impurities like Fe due to their different strain fields (e.g., [49]). The same holds for GBs in $\mathrm{Si}$, which was shown by Chen et al. [50]. In the same publication, it was shown that nearly all GB types might be recombination active if they are heavily contaminated by impurities. In [47], CZ-grown Si with defects was used as model material, and in [43], the model structures have been produced artificially by epitaxial growth of $\mathrm{Si}$ and $\mathrm{Si}-\mathrm{Ge}$ layers on an $\mathrm{n}^{+}$-type (100) Si substrate. Different authors found that particularly SAGBs (tilt angle between approximately $1^{\circ}$ and $3^{\circ}$ ) in $\mathrm{mc}-\mathrm{Si}$ are recombination active, especially if they are decorated by Fe impurities, but no detailed investigation of the SAGBs, i.e., their crystallographic structure, was shown [44], [50]-[52]. Moreover, the scatter in the results is very high. In these works, the type of the GB was defined only by the coincidence site lattice (CSL) or by the inclination of neighboring grains, but not by the orientation of the GB plane relative to the lattice. Due to their strong effect on solar cell performance, the growth and structure of SAGBs and the dislocation distribution and density in VGF ingots were recently investigated in more detail by Miyamura et al. [52] and Oriwol et al. [53], [54]. In [52], it was claimed that at SAGBs, "edge-type dislocations" are responsible for high EBIC contrast. Furthermore, in none of these publications, the exact type(s) of recombination active dislocation(s) could be revealed, and the direct correlation between the increased dark current density $\left(J_{01}\right)$ in mc-Si solar cells and the GB or dislocation types, respectively, has yet to be shown in detail.

Summing up all the above cited knowledge leads to the clear conclusion that iron in different forms is detrimental to mc-Si solar cells. However, there is still a lack of knowledge where at the microscopic scale the recombination takes place. To be precise: Which exactly are the types of crystallographic defects being responsible for strongly enhanced recombination and, thereby, being responsible for inherent material-induced efficiency losses in mc-Si solar cells in the form of enhanced recombination currents? To answer this question, in this paper, we will concentrate on material-induced efficiency-limiting (i.e., highly recombination active) areas in mc-Si solar cells. The problem, which is to solve, is to find precisely the detrimental defects, which only have some nanometer size, on a wafer having an area of $243 \mathrm{~cm}^{2}$ (regarding standard industrial mc-Si solar cells with an edge length of $15.6 \mathrm{~cm}$ ). We will show how it is possible to determine exactly the dominating crystallographic defects across all scales.

\section{EXPERIMENTAL DETAILS}

We used dark lock-in thermography (DLIT) to visualize and analyze the recombinative areas in mc-Si solar cells macroscopically. For the quantitative analysis of local performance losses, the so-called "Local-IV-2" method is used [55], which enables spatially resolved efficiency analysis by calculating the local current-voltage $(I-V)$ characteristics of solar cells. Briefly, the "Local-IV-2" code fits four DLIT images taken at different voltages to a two-diode model enabling to separate the current losses caused mainly by bulk defects (called diffusion current density $J_{\text {diff }}$, with its corresponding saturation current density $J_{01}$ ) from the depletion region current losses $J_{\text {rec }}$ (with its corresponding saturation current density $J_{02}$ ) (see [55]-[57] for details). Since DLIT is limited in its spatial resolution, the areas with increased $J_{01}$ are imaged afterward in high resolution by EBIC imaging in a scanning electron microscope (SEM, JEOL 6400) and during the focused ion beam (FIB) preparation (FEI, Nova Nanolab $600)$ at RT. Based on these EBIC images, selected samples for various (scanning) transmission electron microscopy ((S)TEM) investigations have been prepared by FIB. For performing grain and GB analysis of the selected areas, electron backscattered diffraction (EBSD) measurements were made on an adjacent wafer of the solar cell. The elaborate TEM work was mainly done at a probe $\mathrm{C}_{\mathrm{S}}$-corrected high resolution STEM (FEI TITAN 80-300) in different modes, such as TEM, and selected area diffraction (SAED) and, in particular, high- and low-angle annular dark-field imaging (HAADF, LAADF). Since the acceleration voltage of the TEM amounted to $300 \mathrm{kV}$, we were aware of the possibility of radiation damage during TEM observation. Therefore, the sample regions have been observed at low intensities at higher magnification. We used short exposure times to avoid, e.g., the formation of the so-called rod-like defects (planar faults on $\{113\}$ planes). 


\section{SAMPle ChOICE AND CHARACTERIZATION}

\section{A. High-Precision Selection of Defects With High Recombination Activity}

On a standard industrial full-area Al back contact screenprinted mc-Si solar cell, an area with recombination active defects (increased $J_{01}$, resp. $J_{\text {diff }}$ ) was determined using the Local-IV-2 method [55]. Fig. 1(a) images the diffusion current density $\left(J_{\text {diff }}\right)$ at $550 \mathrm{mV}$ of this solar cell. The bright areas are typical clusters with a high density of recombination active defects (i.e., with high $J_{\text {diff }}$ ), one is marked by a rectangle. Fig. 1(b) shows, as countercheck, the $J_{\text {rec }}$ image at $550 \mathrm{mV}$ where no signal is visible at this position. Hence, here only strong recombination active bulk defects lead to efficiency losses of the solar cell. This area was cut out. The small piece with the size of $15.3 \mathrm{~mm} \times 17.3 \mathrm{~mm}$ was then analyzed again with the Local-IV2 method. In Fig. 1(c), the image of the diffusion current density $J_{\text {diff }}(550 \mathrm{mV})$ of the small sample is shown, which was calculated from the DLIT data [55]. A clear structure of high $J_{\text {diff }}$ areas is visible, which corresponds to the structure of low EBIC signals as can be seen in Fig. 1(e). The EBIC image in Fig. 1(e) was taken at $30 \mathrm{kV}$; hence, the penetration depth of the electron beam is about $8 \mu \mathrm{m}$, therefore mainly bulk defects are imaged. Each dark line in Fig. 1(e) is a recombination active extended defect; mostly an SAGB within a large grain, which is a so-called subgrain structure. Exactly at the positions of high $J_{\text {diff }}$ and low EBIC signals, the efficiency of the cell is significantly lower compared with the good areas, demonstrated by the calculated efficiency image in Fig. 1(d). At the positions marked by the circles in Fig. 1(c)-(f), the efficiency is only about $13 \%$ (abs.) or even lower, whereas the good areas of the cell show efficiency values of up to $17 \%$ (abs.). Going into detail, at all recombination active defects, i.e., dark lines in Fig. 1(e), high $J_{\text {diff }}$ is observed.

To evaluate the correlation between the GB structure and the structure of the recombination active defects, an EBSD map was taken at the direct adjacent wafer of the investigated solar cell exactly at the position where the DLIT and EBIC analysis have been performed on the solar cell. The distance between the wafers is about $200 \mu \mathrm{m}$; hence, it can be assumed that the grain structure is very similar. The step width of the EBSD scan was $50 \mu \mathrm{m}$. In Fig. 1(f), the inverse pole figure representation of the EBSD scan showing different grain orientations in color code is given. Fig. 1(f) also includes the GBs evaluated by the EBSD system; in black, CSL GBs are displayed (approximately $72 \%$ of all detected GBs), and in red, SAGBs (approximately $28 \%$ of all detected GBs) are shown, whereas the CSL GB separate two grains with distinctly different orientation from each other, SAGBs are found within the large grains, and are also called subgrain structures. Most of the CSL GBs [black lines in Fig. 1(f)] found in this sample are $\Sigma 3\left(\langle 111\rangle 70.53^{\circ}{ }_{-}\right.$ twin GBs) and $\Sigma 9$ GBs (in sum $89 \%$ ), and only a small share are $\Sigma 5, \Sigma 27$, and $\Sigma 47$ GBs (in sum $11 \%$ ). The lines of the CLS GBs are also overlayed in the EBIC image in Fig. 1(e) to give an orientation of the location of the grains in the sample. Note that for better visibility and for distinguish them from the dark EBIC contrast lines, they are colored white [in contrast with Fig. 1(f), where they are black]. There is no correlation between the CSL
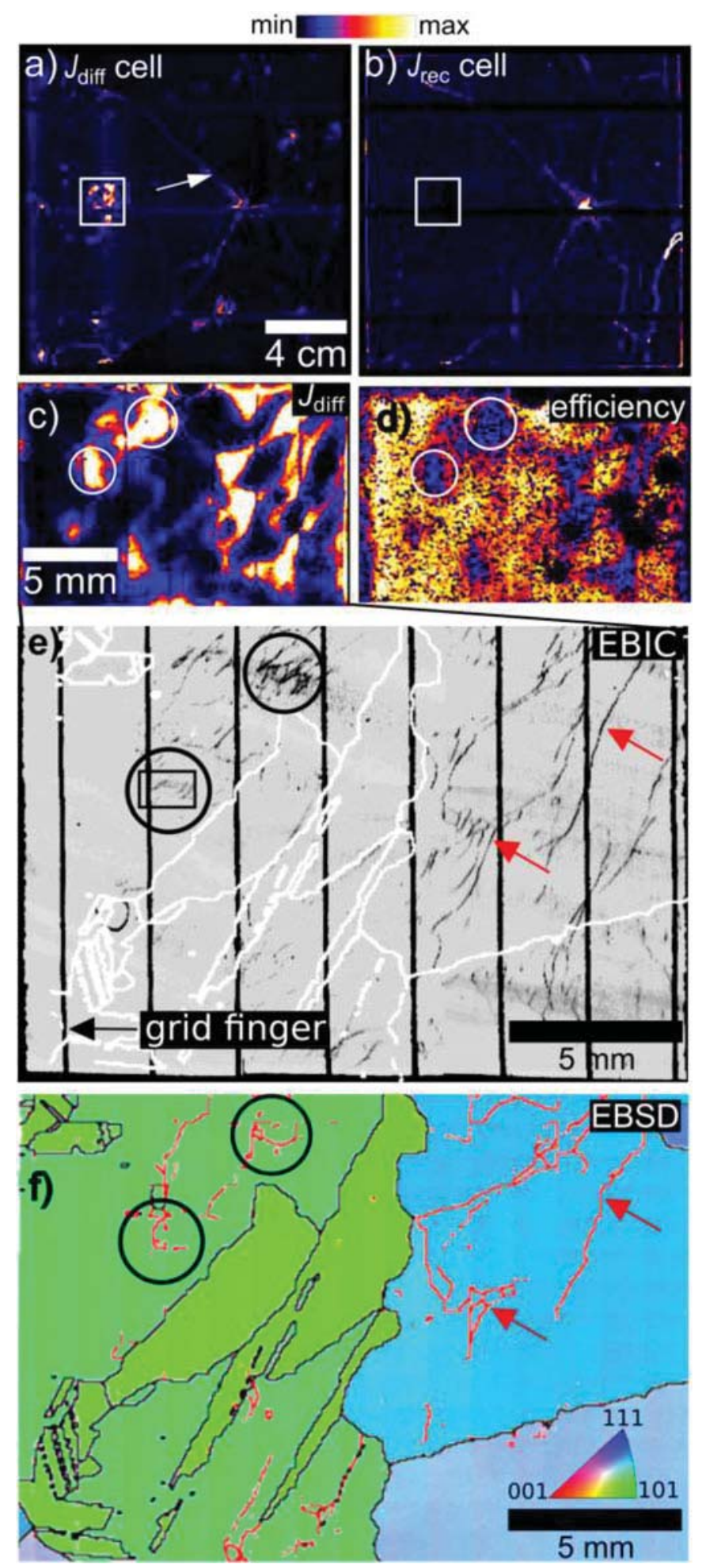

Fig. 1. Images (a)-(d) refer to the color scale at the top. (a) $J_{\text {diff }}(550 \mathrm{mV})$ of an mc-Si solar cell area with increased $J_{\text {diff }}$ is marked by the rectangle. (b) $J_{\text {rec }}(550 \mathrm{mV})$ image of the same cell. (c) Magnified $J_{\text {diff }}$ image at the position of the rectangle in (a) (cut-out sample). Scaling for (a) to (c): $\mathrm{min}=0 \mathrm{~mA} / \mathrm{cm}^{2}$, $\max =3.5 \times 10^{-2} \mathrm{~mA} / \mathrm{cm}^{2}$. (d) Efficiency image corresponding to (c), scaling from $12 \%$ to $17 \%$. (e) EBIC image taken at $30 \mathrm{kV}$ of the corresponding area is shown, and the white lines are overlaid CSL GBs [see (f)]. (f) EBSD map containing CSL GBs [black lines = white lines in (e)] and SAGBs (red lines). In (a), a crack in the solar cell is marked exemplarily by the arrow. The cracks do not influence the results. Red arrows in (e) and (f) point exemplarily to SAGBs (for an explanation of circles, see the text). 


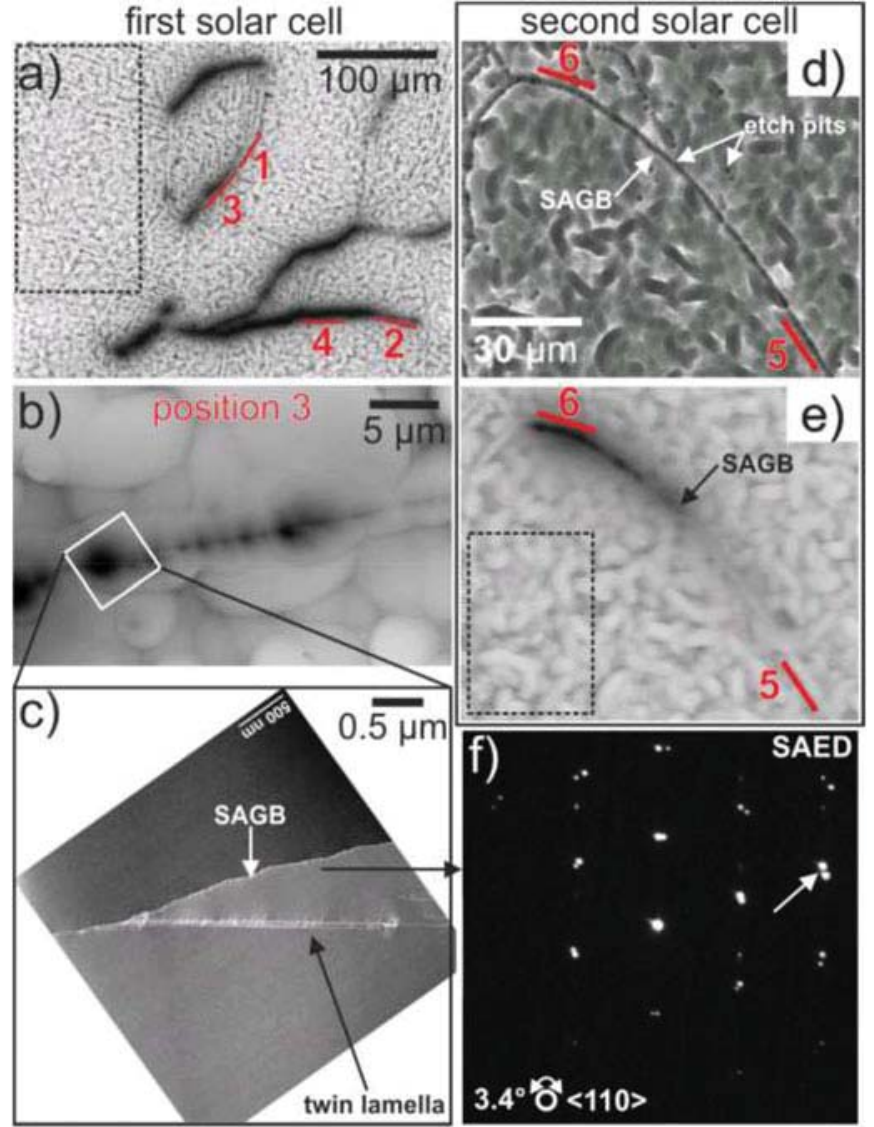

Fig. 2. (a) Detailed EBIC image out of the rectangle in Fig. 1(e), where the four position of GBs with different EBIC signal strength used for FIB preparation are marked. The dotted rectangle is used for EBIC analysis (see Section V). (b) Highly magnified EBIC image of position 3. (c) LAADF image of the FIB sample made from one part of position 3. (d) SEM image of a further SAGB of another sample (second solar cell). (e) Corresponding EBIC image of (d). (f) Corresponding SAED pattern of the SAGB.

GBs [black lines in (f), white lines in (e)] and the recombination active defects. However, most of the small angle GBs correlate with recombination active defects. Only some examples are marked in Fig. 1(e) and (f) by arrows to keep the figures still readily comprehensible. At some recombination active defect structures, no GBs are found, which might be due to the EBSD scan resolution of $50 \mu \mathrm{m}$. Concluding the results of Fig. 1, we may say that: There is a direct lateral correlation between increased $J_{\text {diff }}$ in (c), lower efficiency in (d), dark EBIC features in (e), and SAGBs in (f), which is exemplarily emphasized by the two circles in the denoted images each.

Detailed DLIT, EBIC, and STEM investigations have been performed on another solar cell sample as well (see Section III-B for details). In Fig. 2, EBIC images of both samples are shown. The first solar cell sample (left side in Fig. 2) is that marked by a rectangle in Fig. 1(e), whereas the second solar cell sample (right side in Fig. 2) stems from another cell but showed the same behavior. In Fig. 2(a), a detailed EBIC image out of the high $J_{01}$-area [marked by the rectangle in Fig. 1(e)] is given. Already at this magnification, it is obvious that the EBIC contrast at different SAGBs and even within one and the same SAGB is different. In the case of this sample from the first solar cell, at four positions showing different EBIC signals, planar FIB lamellae (i.e., parallel to the surface) have been prepared from a depth of about $1 \mu \mathrm{m}$ below the solar cells surface and thinned down to a thickness of about 100 to $50 \mathrm{~nm}$, which is necessary for STEM investigations. The EBIC contrast is weakest at position 1 and increases up to position 4, as shown in Fig. 2(a). Note that FIB lamellae from positions 1, 3 and 2, 4 belong to the same GB, respectively, but to different recombination activities. The highly magnified EBIC image of position 3 [taken at an acceleration voltage of $30 \mathrm{kV}$, and displayed in Fig. 2(b)] reveals a very inhomogeneous EBIC contrast distribution along the GB. The EBIC contrast is point like and shows very different values, the points correlate with etch pits, which are frequently found in the acidic texturized mc-Si solar cells [58].

Wrapping up the results so far, SAGBs showing significant higher $J_{01}\left(J_{\text {diff }}\right)$, which is subsequently reducing locally the solar cell efficiency by up to approximately 4\% (absolute) [59], have been found in our sample. Four positions with recombination active GBs have been particularly considered. The four chosen positions show different strong locally (along the SAGB) distributed recombination, which was revealed by EBIC images [see Fig. 2(b)].

\section{B. STEM Investigations}

To reveal the type of the GBs and their detailed crystallographic structure, STEM investigation have been performed at the positions shown in Fig. 2. In addition to the above described four positions from the first solar cell, another two samples have been precharacterized and prepared in the same way. These two samples, named position 5 and 6 , have been taken from a stateof-the-art industrial mc-Si solar cell randomly chosen from our shelf. This was done, therefore, to prove that the findings in our first solar cell are not singular and accidental results but are rather generally meaningful for this type of solar cells.

First, the findings in solar cell one are considered: In Fig. 2(c), an LAADF image out of the left part of the GB of position 3 from Fig. 2(b) is shown. The image reveals that the GB is not straight but contains kinks. Some defects evolve from the GB, which have been identified to be twin lamellae (see below for details). At the GB of position 3, an SAED pattern was taken, which is shown in Fig. 2(f). The split of the spots (see arrow) reveals that the GB is an SAGB with a tilt angle of about $3.4^{\circ}$ around the $\langle 110\rangle$ direction. This tilt angle holds for the whole SAGB (except the joining regions of the twin lamellae), but obviously, the recombination activity strongly depends on the SAGB orientation. At all other positions shown in Fig. 2(a), the GBs have also been identified to be SAGBs with tilt angle of about $3^{\circ}$, respectively.

In Fig. 2(d), an SEM, and, in (e), an EBIC image of a part of solar cell two are shown, respectively. This sample has been taken from a part of a solar cell being comparable with the first one (as shown in Fig. 1). Hence, it was investigated by DLIT and EBIC as well, revealing the same properties (i.e., SAGB, high $J_{01}$, locally decreased efficiency, high EBIC contrast) as found for the positions 1 to 4 and presented in Figs. 1 and 2. Planar FIB preparations have been done in two different positions of the GB 

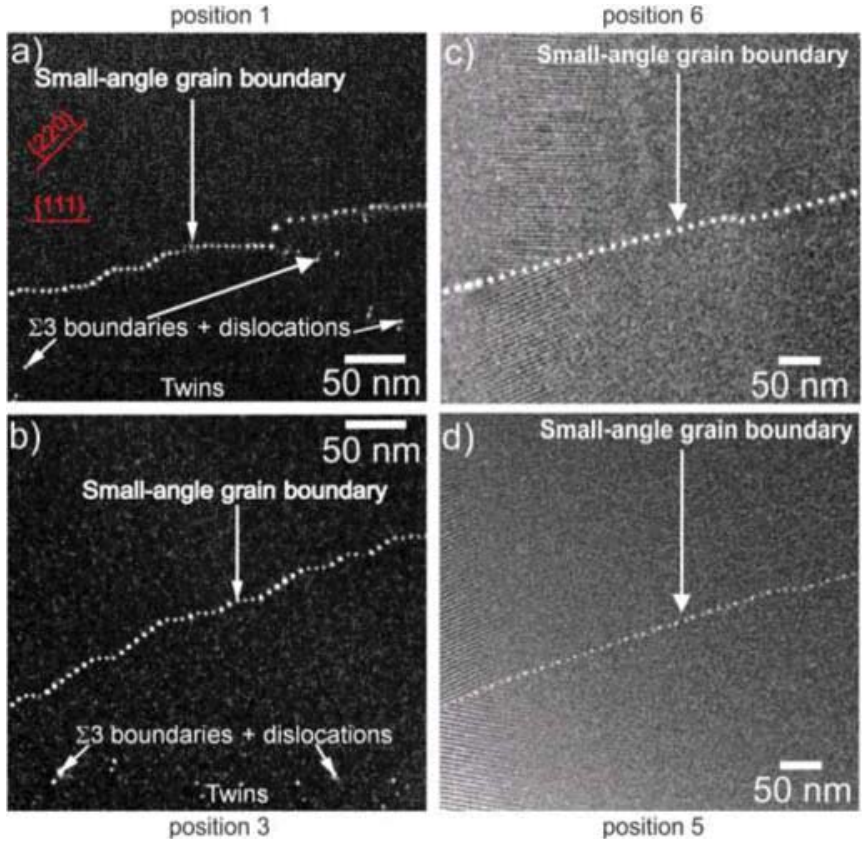

Fig. 3. LAADF-STEM images of four FIB lamellae (out of the six planar lamellae described above). (a) STEM image of position 1 having a low EBIC contrast. (b) STEM image of position 3 (strong EBIC contrast). (c) and (d) STEM images of positions 6 (strong EBIC contrast) and 5 (no EBIC contrast) are shown, respectively. At all SAGBs bright spots are detectable by LAADFSTEM, each bright spot being a dislocation. Plane orientations for (a) and (b) are given in (a).

of the second solar cell, which are marked in Fig. 2(d) and (e) by the red lines. One FIB lamella was prepared in the position of the GB showing no EBIC contrast (position 5), and a second one was prepared at a part of the GB, which shows very high EBIC contrast (position 6). The SEM image displayed in Fig. 2(d) reveals a very high density of etch pits at the SAGB, which is also true for the SAGBs of the sample from the first solar cell (no SEM image shown here). Some etch pits are also found in the vicinity of the SAGB [see arrows in Fig. 2(d)]. The SAGB is not straight but shows a strong bend, i.e., the angle of the SAGB plane is changing with respect to the grain orientation. The EBIC image in Fig. 2(e) reveals strong recombination activity at the upper part (position 6), and low to none recombination activity at the lower part (position 5) of the SAGB. The structure of the EBIC signals in Fig. 2(a) and (e) look qualitatively very similar: There are some points of stronger contrast along the SAGB, the EBIC contrast changes along the SAGB, and at both SAGBs, a high density of etch pits is found.

Fig. 3 shows LAADF-STEM images of positions 1, 3, 5, and 6. The images of positions 1 and 3 in Fig. 3(a) and (b) are displayed in the correct orientation to each other, which reveals that the SAGBs change their inclination in relation to the $\{111\}$ planes. Note that positions 1 and 3, as well as positions 5 and 6 belong to one and the same SAGB, respectively. In all pictures shown in Fig. 3, single point-like bright contrasts features are visible at the SAGBs in high density. These bright spots are attributed to different types of dislocations (see Section IV below for details). The SAGB in positions 1 and 3 shows much more steps compared with the SAGB in positions 5 and 6 . The density of spots is approximately the same for each SAGB, but there is

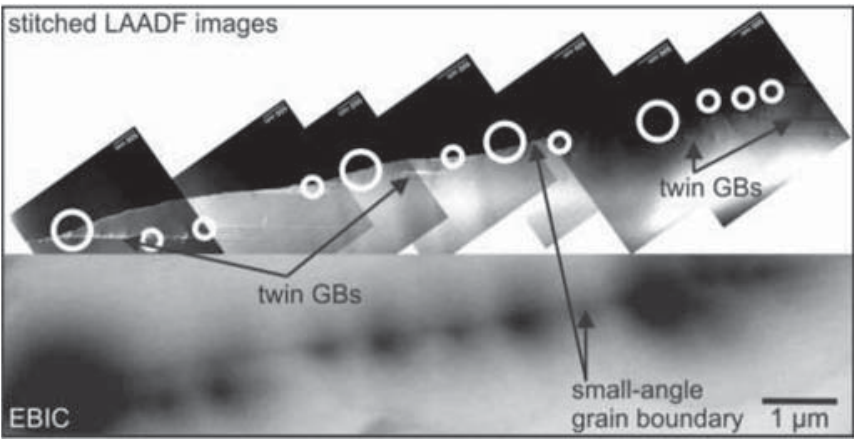

Fig. 4. Stitched LAADF STEM images of the SAGB of position 3 and the corresponding EBIC image.

no correlation between EBIC contrast and overall spot density (see details in Section V, and Fig. 8). A difference between positions 1, 3 and 5, 6 is found in the vicinity of the SAGBs. In positions 1 and 3, some twin GBs and a small number of single dislocations have been found [see Figs. 2(c), 3(a) and (b), and 4], which is not the case in positions 5 and 6 . Due to the fact that in the direct vicinity of the SAGBs in positions 1 and 3 twin boundaries and single dislocation have been found, it must be proven where exactly the EBIC contrast occurs. In Fig. 4, stitched LAADF-STEM images of an approximately $10 \mu \mathrm{m}$ long part of the SAGB of position 3 are shown. Below the LAADF images, the corresponding EBIC image is given. The white circles mark the approximate location of the dark EBIC spots at the SAGB, the size of the circles correspond to the approximate strength of the EBIC contrast. The comparison of both images reveals clearly that mainly at the SAGB high EBIC contrast (i.e., high recombination activity) can be detected, but there is almost no increased EBIC contrast at the twin GBs.

\section{Detailed STEM ANAlysis of the Dislocations AT THE SMALL-ANGLE GRAIN BOUNDARY}

For the six SAGB positions prepared by FIB (cf., Fig. 2), detailed STEM investigations have been done. The first goal was to reveal the types of dislocations found at the SAGBs, and the second goal was to look for impurity atoms in the SAGBs (e.g., iron). The twin GBs and single dislocations found in the vicinity of the SAGB have been investigated in detail as well. However, since they do not show any recombination activity, they are not in the focus of this paper. Briefly, we found twin GBs ( $\Sigma 3\{111\})$, twin grains, and intrinsic stacking faults (SFs), which are bordered by partial dislocations (pd). It is well known from the literature that $\Sigma 3$ GB twins are not harmful to solar cells [50], [51], which is confirmed by our studies. Obviously, SFs and pd are also not harmful concerning high recombination activity. All SAGBs in the FIB lamellae 1 to 6 have been investigated by STEM in detail. The white spots, which are present at each SAGBs in Fig. 3 have been identified to be dislocations. However, we have the following question: Which kinds of dislocations exist there? In each position, a very large number of dislocations have been investigated by LAADFSTEM down to the atomic scale to determine the dislocation types. For example, in Figs. 5 and 6, detailed images of the SAGBs showing different EBIC contrasts are displayed, and 


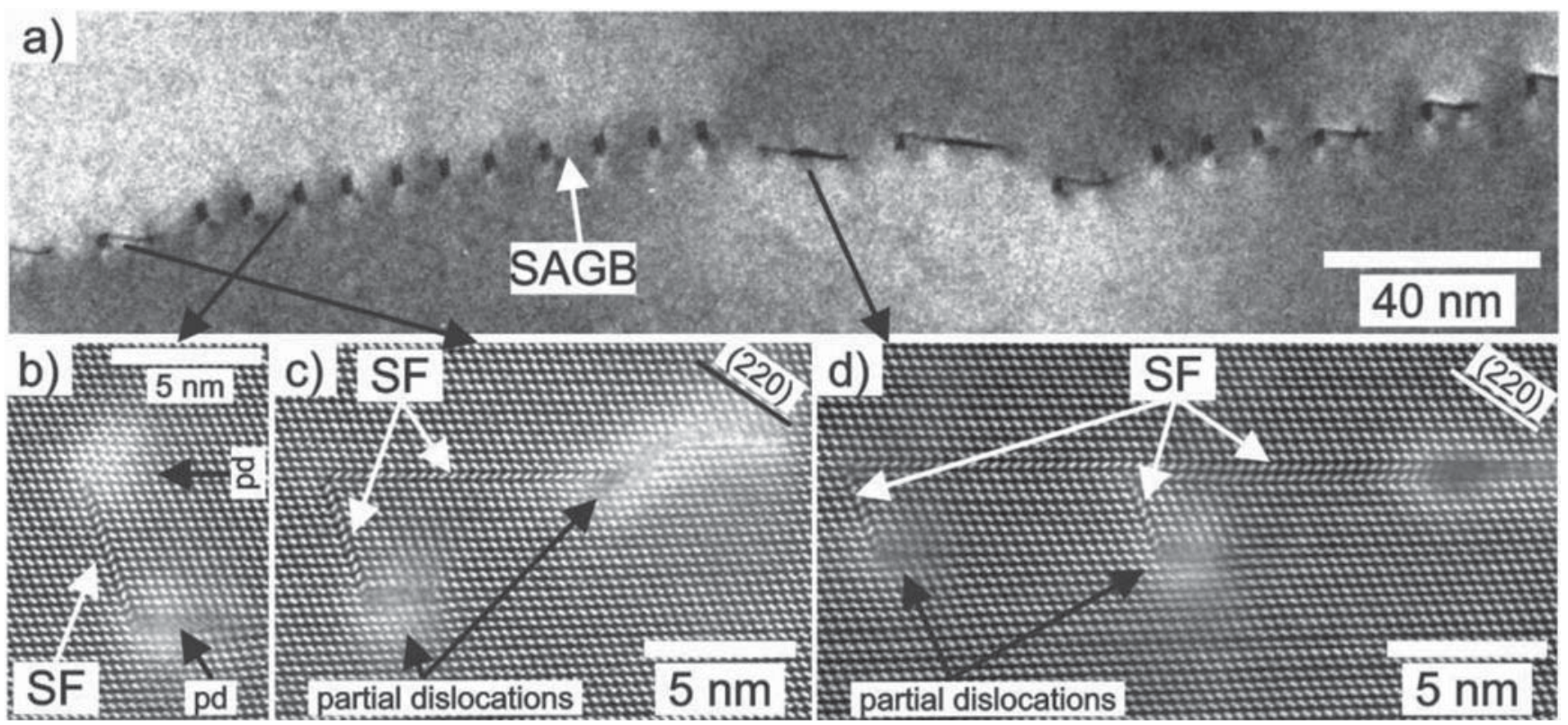

Fig. 5. Arrangement of dislocations at the SAGB of position 5 (no EBIC contrast). (a) Bright-field TEM image showing different types of split dislocations. (b)-(d) LAADF-STEM images of three types of split dislocations (description in the text). In all images, the crystal lattice including the defects is seen in the $\langle 110\rangle$ sample orientation.

related to detailed images of the dislocations found, respectively. In Fig. 5, the dislocations at the SAGB of position 5 with no EBIC contrast are shown, whereas in Fig. 6, the dislocations found at positions 1 and 6 with higher EBIC contrasts are given.

An overview of the SAGB of position 5 is shown in Fig. 5(a). It is a bright-field TEM image with $\langle 110\rangle$ crystal orientation. Please note that within the whole part of the SAGB in position 5, which was caught in the FIB lamella (approximately $10 \mu \mathrm{m}$ in length), all dislocations are split and are characterized by SF and dislocations at their borders running in $\langle 110\rangle$ orientations. With respect to the Burgers vectors, the splitting can be expressed in general as: $\frac{a}{2}\left\langle\begin{array}{lll}1 & 1 & 0\end{array} \rightarrow \frac{a}{6}\langle 112\rangle+\frac{a}{6}\langle 11 \overline{2}\rangle\right.$. The two latter ones are referred as to Shockley pd ( $a=$ lattice constant).

Three specific types of corresponding dislocations could be identified in this SAGB region. Type 1, exemplarily shown in Fig. 5(b), corresponds to the simple split $60^{\circ}$ dislocation with an SF bordered by pd, respectively. Dislocation type 2 is represented in Fig. 5(c). The defect is composed of two intrinsic SFs confining an angle of $71^{\circ}$. They are bordered by two pd of the type $\frac{a}{6}\langle 112\rangle\left(30^{\circ}\right.$ Shockley partial at the lower left, $90^{\circ}$ Shockley partial at the upper right). The line defect at the intersection of the two SFs has a Burgers vector of $\frac{a}{6}\langle 011\rangle$ size. The third type consists of a complex configuration of a horizontal SF with two outgoing SFs (being bordered by partial $30^{\circ}$ dislocations) and an extrinsic SF (being bordered by a pd (right)). An example of this defect type is displayed in Fig. 5(d).

A different structure and arrangement of dislocations is found in the SAGB at position 6, which is characterized by a strong EBIC contrast. Fig. 6 shows, for comparison, crystal lattice images (LAADF-STEM) of the weak-EBIC contrast position 1 [see Fig. 6(a)], similar to the case shown in Fig. 5, and of strongEBIC contrast at position 6 [see Fig. 6(b)]. For the latter case, the boundary dislocations having a distance of about $15 \mathrm{~nm}$ and are not split (nondissociated). Only a small fraction (approximately $5 \%$ ) of the dislocations found in position 6 have been identified to be split defects with pd and SFs, all of them are located at the kinks of the SAGB [a kink of the SAGB at position 6 is shown for example in Fig. 3(c)].

\section{A. Burgers Vector Analysis of Boundary Dislocations at Positions 1 and 6}

In order to determine the types of the nonsplit boundary dislocations, we analyzed the corresponding Burgers vector. As an example, Fig. 7 shows the SAGB for the positions 1 (a) and 6 (b), respectively. For the analysis, we used high-resolution HAADF micrographs as demonstrated in Fig. 7(a). Here, the crystal lattice (Si dumbbells) is seen in $[\overline{1} 10]$ orientation, which is the direction pointing into the paper plane. The SAGB is marked by a dashed line in Fig. 7(a). Fig. 7(b) shows the same type of dislocation received from position 6 . Here, the dislocation core is marked by a circle. For better visibility of the atom position the image in (b) was converted and its contrast was manipulated, which can be seen in (c). The Burgers circuit ABCDE is drawn around a boundary dislocation core in Fig. 7(b). The closure vector $E A$ yields a Burgers vector $\vec{b}$ of $\frac{\vec{a}}{2}[110]$ and the gliding plane $\vec{v} \times \vec{b}=(001)$, which is typical for Lomer dislocations [60]. The dislocation line $\vec{v}$ itself points to [110] as it was found for the dislocation in Fig. 7(a). The image in Fig. 7(b) was taken along the [110] crystallographic direction, with the dislocation line being in edge-on configuration. The core of the dislocation shows the structure of a classic Lomer dislocation according to Bourret et al. [60]. In all investigated cases, the Lomer dislocations are undissociated with a Burgers vector of $\vec{b}=\frac{\vec{a}}{2}\langle 110\rangle$. 

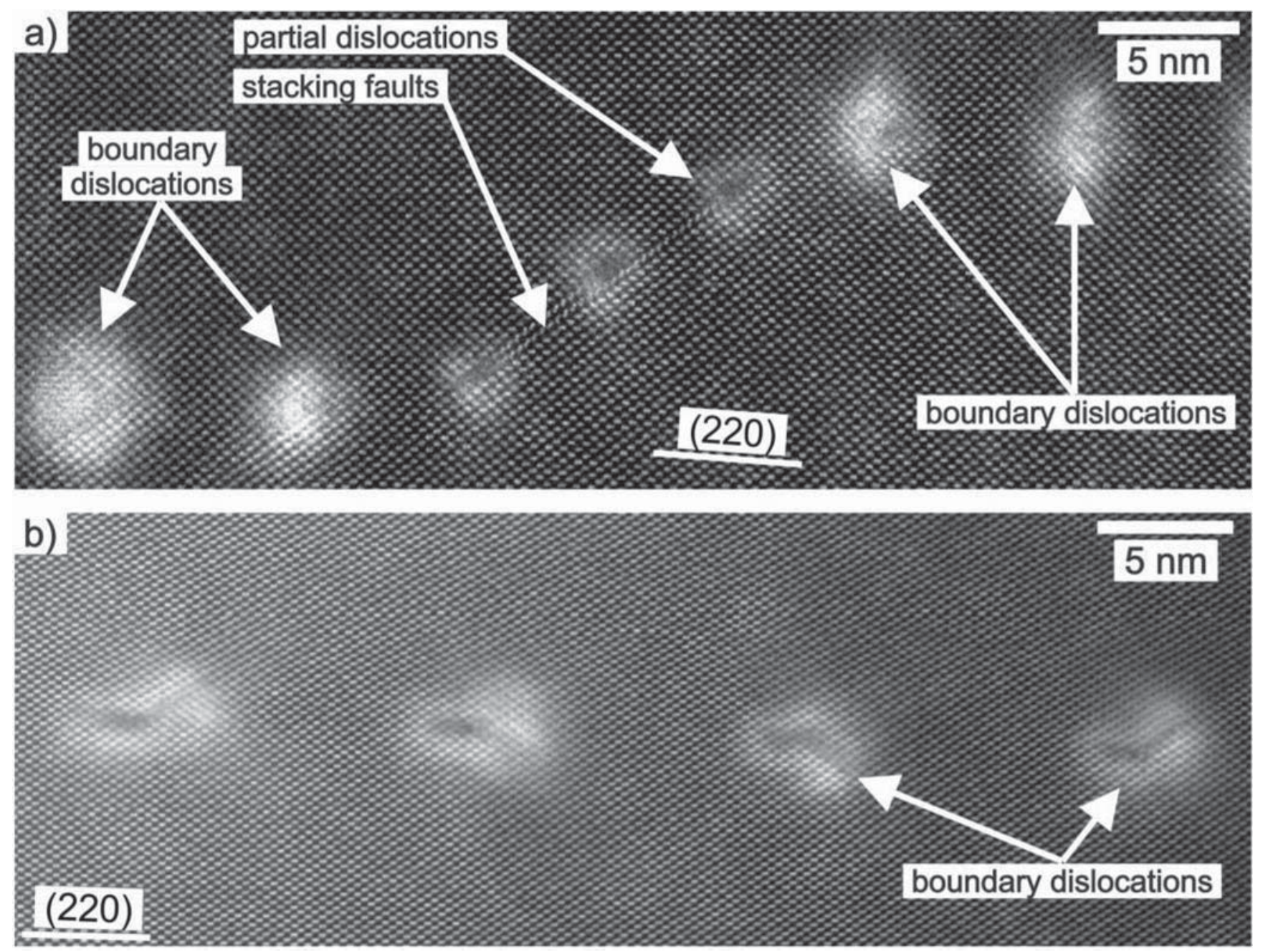

Fig. 6. LAADF-STEM images of different boundary regions including different types of dislocations. (a) SAGB correlating with position 1 (low EBIC contrast). (b) SAGB correlating with position 6 (high EBIC contrast), whereas the SAGB in (b) is characterized by nonsplit Lomer dislocations, and region (a) includes split dislocations, as well as some nonsplit dislocations. In both images, the crystal lattice including the defects is seen in a $\langle 110\rangle$ sample orientation.

\section{B. Lomer Dislocations and Lomer-Cottrell Lock}

In [52], the dislocations responsible for high recombination activity at the SAGBs have been shown to be edge dislocations. Perfect (undissociated) Lomer dislocations are edge dislocations lying along [011] and having a (100) slip plane [60]. They may be formed, e.g., in plastic deformation experiments by the reaction of two parallel dislocations lying in different slip planes. The (100) slip plane of the Lomer dislocation is not the common (111) slip plane, therefore Lomer dislocations are considered to be quite immobile. Cottrell has proposed in 1952 that they may become very immobile if they dissociate into a Lomer-Cottrell lock [61]. Sekiguchi and Sumino [62] have found D1 and D2 luminescence in regions of plastically deformed $\mathrm{Si}$ where plural slip lines intersect each other, and they suspected that LomerCottrell locks are responsible for this luminescence. Nonsplit Lomer dislocations in mc-Si materials can be regarded as grownin, although they also may results from plastic deformation processes during cool down of the block. Miyamura suspected that the deep levels responsible for the recombination activity of the edge dislocations found in [62] are the same as the ones responsible for the D1 and D2 luminescence; therefore, they concluded that these edge dislocations could be Lomer-Cottrell locks. This interpretation was followed by Oriwol [54]. In all these publications, no direct proof by TEM or other techniques of the dislocation being responsible for high recombination was shown.

However, all our findings clearly exhibit that perfect Lomer dislocations and not Lomer-Cottrell locks play a major role for recombination at subgrain structures like SAGBs in mc-Si. This fits to all our samples, which are very typical samples, investigated during our study. It is important to clarify this problem to be consistent in the scientific discussion. Even Bourret et al., have found that grown-in Lomer dislocations are undissociated and that the formation of Lomer-Cottrell locks is improbable [60]. Furthermore, in contrast with a Lomer dislocation, a Lomer-Cottrell lock shows a symmetric arrangement of the atoms in the dislocation core [60]. We could not observe this in our samples. So far, in all our samples, we only detect perfect (undissociated) Lomer dislocations that are responsible for the high recombination activity. 


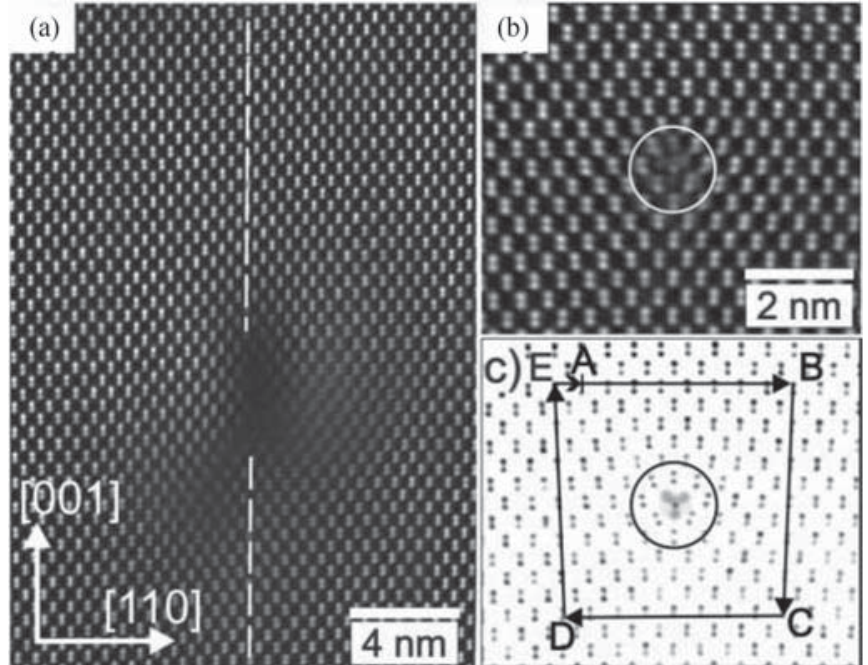

Fig. 7. Determination of the Burgers vector of the boundary defect identified as Lomer dislocation. (a) Typical HAADF micrograph of a dislocation from position 1 , where the GB is marked by a dashed line. The lattice structure ( $\mathrm{S}$ dumbbells) is seen in the $\left[\begin{array}{lll}\overline{1} & 1 & 0\end{array}\right]$ orientation (into the paper plane). (b) HAADF micrograph of the same type of dislocation from position 6; its core is marked by a circle. As in (a), the dislocation line has a $\left[\begin{array}{lll}\overline{1} & 1 & 0\end{array}\right]$ direction. (c) Construction of a Burgers circuit (A-B-C-D-E) in the point lattice. The image is received by contrast manipulations of the original HAADF image from (b). The Burgers vector corresponds to the distance $\mathrm{E}-\mathrm{A}$.

\section{Correlation Between lomer Dislocation Density AND RECOMBINATION ACTIVITY}

In the previous section, the defects at the SAGBs have been identified being Lomer dislocations, pd, and SFs. How are these different types of defects related to the recombination activity? As shown in Fig. 2, the recombination activity of the SAGBs is locally varying. It turned out that at SAGBs of positions having an increased EBIC contrast, Lomer dislocations have been found, whereas at parts of the SAGB showing no EBIC contrast (see Figs. 5 and 6) and no Lomer dislocations, but instead pd and SFs in different configurations, have been observed. By, respectively, counting and recording the locations of the different dislocation types at each SAGB position investigated, it was found that the recombination activity and the density of Lomer dislocations correlate. In Fig. 8, the EBIC contrast is plotted via the total dislocation density (marked by black triangles) and the Lomer dislocation density (marked by red dots) for each of the six positions, respectively. Note that EBIC was measured at RT, and the contrast at the SAGB $C_{\mathrm{GB}}$ is defined as $C_{\mathrm{GB}}=\left(I_{0}-I_{\mathrm{GB}}\right) / I_{0}$ [63], with $I_{0}$ being the EBIC signal (gray value in our case) far away from the SAGBs, and $I_{\mathrm{GB}}$ being the signal averaged along the SAGB parts corresponding to the positions given in Fig. 2. The contrast values have been determined using the original EBIC images (not shown here, the EBIC images shown in this paper are processed for the sake of better visibility). Due to the fact that two EBIC images with different contrast settings are compared, both images have been normalized to the maximum gray value, respectively. Since the EBIC signal far away from the GBs is influenced by different effects due to the roughness of the sample's surface, we determined $I_{0}$ by calculating the mean gray value of the dotted rectangles (in the original images)

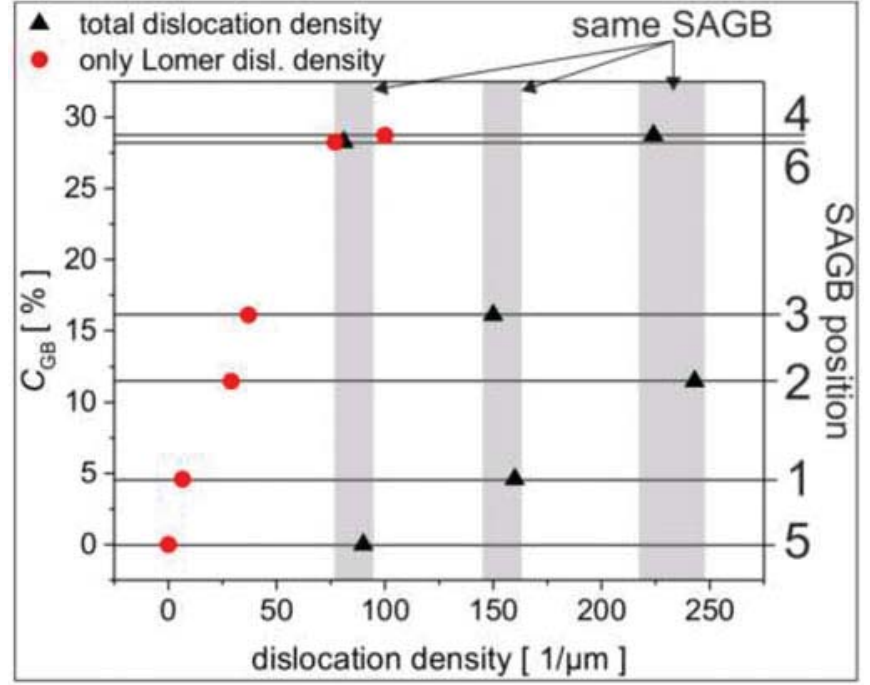

Fig. 8. Averaged EBIC contrast $C_{\mathrm{GB}}$ at the SAGBs, depending on the averaged Lomer dislocation density along the length of each SAGB position 1 to 6 (red dots). Black triangles: $C_{\mathrm{GB}}$ versus overall dislocation density for each position. Data points on the same line belong to the same SAGB position named on the right. Black triangles within a gray stripe belong to one and the same $\mathrm{SAGB}$, respectively.

TABLE I

EBIC CONTRAST AND DisLOCATION DENSITY DATA (POSITIONS FROM THE SAME SAGB HAVE SAME BACKGROUND COLOR)

\begin{tabular}{lcccc}
\hline \hline Position & $\begin{array}{c}\text { EBIC } \\
\text { contrast } \\
C_{\mathrm{GB}} \text { in } \%\end{array}$ & $\begin{array}{c}\text { all } \\
\text { dislocations } \\
\text { density } \\
(1 / \mu \mathrm{m})\end{array}$ & $\begin{array}{c}\text { Lomer } \\
\text { dislocations } \\
\text { density } \\
(1 / \mu \mathrm{m})\end{array}$ & $\begin{array}{c}\text { Lomer } \\
\text { dislocation } \\
\text { percentage } \\
(\%)\end{array}$ \\
\hline 1 & 5 & 160 & 7 & 4 \\
2 & 12 & 243 & 29 & 12 \\
3 & 16 & 150 & 37 & 25 \\
4 & 29 & 224 & 100 & 45 \\
5 & 0 & 90 & 0 & 0 \\
6 & 28 & 81 & 77 & 95 \\
\hline \hline
\end{tabular}

marked in Fig. 2(a) and (e), respectively, which contain no recombination active GB.

Note that the dislocation densities shown in Fig. 8 are averaged along the length of the respective SAGB position taken from several STEM images. It was found that in each SAGB, the overall dislocation density is more or less constant, which is highlighted by the vertical gray stripes in Fig. 8, and can also be seen in Table I. The overall dislocation density ranges between $90 \mu \mathrm{m}^{-1}$ and about $240 \mu \mathrm{m}^{-1}$. The percentage share of Lomer dislocations is different for each position and does only show a clear correlation with the EBIC contrast at values up to a share of about $50 \%$. This leads to the assumption that only the absolute number of Lomer dislocations per SAGB length is significant for the recombination activity. In Table I, all data mentioned above is wrapped up for a better overview.

There is a strong correlation between the Lomer dislocation density and EBIC contrast, which is shown by the red dots in Fig. 8. The higher the density of Lomer dislocations, the higher is the EBIC contrast. At high densities of Lomer dislocations, the EBIC contrast starts to saturate, which was also found in principle in [48]. This saturation might be due to a lower degree 


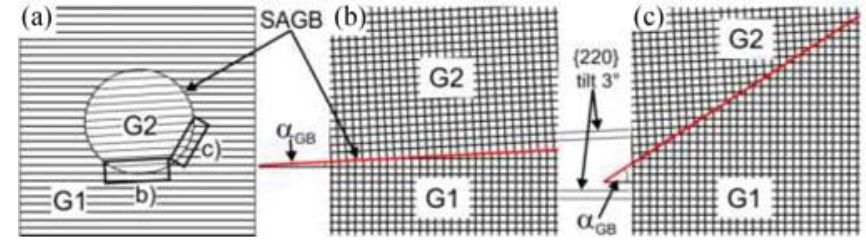

Fig. 9. (a) Sketch of a subgrain G2 within a grain G1. The azimuthal orientation of the boundary is changing along the circle [red line in (b) and (c)]. The rotation angle $\mathrm{a}_{\mathrm{GB}}$ between the two grains amounts to $3^{\circ}$. (b) SAGB has a small azimuthal angle to the $\{220\}$ lattice planes of G1 resulting in high Lomer dislocation density. (c) SAGB has a large angle to the $\{220\}$ lattice planes of G1 resulting in a low density of Lomer dislocation.

of contamination of the Lomer dislocations if they are present in high density or due to the very high recombination velocity at the dislocations, which cannot be resolved by the EBIC measurement. The average density of Lomer dislocation at the different SAGB positions accounts from approximately $7 \mu \mathrm{m}^{-1}$ at position 1 showing the weakest EBIC contrast of about 5\%, to approximately $100 \mu \mathrm{m}^{-1}$ at position 4 showing the highest EBIC contrast of about $29 \%$. In position 5, where no Lomer dislocation could be found, indeed no EBIC contrast was detectable (see Fig. 2 and Table I). Furthermore, there is no correlation between the overall dislocation density and the EBIC contrast $C_{\mathrm{GB}}$ at the SAGBs, which is proved by the arbitrary distribution of the EBIC contrast versus overall dislocation density represented by the black triangles displayed in Fig. 8 .

\section{A. Orientation of the Small-Angle Grain Boundaries}

In the investigated SAGBs, the density of nonsplit Lomer dislocations and split dislocations containing partials and SFs depends on the rotation angle between the crystal lattices of the so-called main grain and the subgrains, which are separated by the SAGB. In Fig. 2(d) and (e), we demonstrate that the SAGB is curved and that the EBIC contrast along this specific SAGB line is changing. We would like to emphasize that along such curves the crystal structure of the SAGB is varying, although the rotation angle between these two adjacent grains does not change. This is schematically demonstrated in Fig. 9 to support the following discussion.

In Fig. 9(a), an idealized circular subgrain (G2) is embedded within a large grain $(\mathrm{G} 1)$. In this specific example, the rotation between grain $\mathrm{G} 1$ and $\mathrm{G} 2$ amounts to about $3^{\circ}$ similar to the experimental situation. This is indicated by the line system, which might represent the crystal lattice, e.g., the $\{220\}$ lattice planes. Along the circular SAGB, the crystallography of the boundary is varying. Fig. 9(b) represents the specific situation at an azimuthal boundary condition corresponding to position 6 . The crystal structure at the boundary is idealized by a rectangular network; the rotation angle between G1 and G2 is indicated by $\alpha_{\mathrm{GB}}$, and the azimuthal inclination is indicated by a red line. In our experiments, we found, at such a lattice inclination, a high density of nonsplit Lomer dislocation as well as high EBIC contrast. Fig. 9(c) sketches a different crystallographic situation, which refers to a situation found in position 5 (no EBIC contrast). Here, the azimuth of the boundary is rotated by about $30^{\circ}$ with respect to Fig. 9(b). In the related experiments, the den- sity of Lomer dislocations is lowered; however, the number of split dislocation (pd and SFs) is increased. This is accompanied by decreased EBIC contrast.

The ratio between nonsplit Lomer dislocations and split dislocations depends on this inclination angle and might be the results of a crystallization process to minimize the GB energy.

However, the building mechanisms of the different dislocation structures at SAGBs in Si are not yet known in detail. Some recent results about the formation of dislocation clusters in VGFgrown Si blocks for solar cells can be found, for instance, in [53] and [54].

\section{DISCUSSION AND CONCLUSION}

A clear correlation between the density of Lomer dislocations at SAGBs and their EBIC contrast was found in the presented study. However, due to the complex structure of GBs, dislocations, and extended defects in silicon, it is still possible that other defects might be identified because they are also the cause of recombination activity. Nevertheless, in all samples we investigated so far, only Lomer dislocations have been detected at the recombination active positions of the SAGBs and no LomerCottrell locks.

Donolato defined the normalized recombination activity $\Gamma$ of dislocations as the influence of the dislocation density on the minority carrier diffusion length at the dislocations compared with the background diffusion length [64]. This was later proven experimentally by Rinio [5], who showed a correlation between the internal quantum efficiency and the dislocation density in connection with the recombination activity $\Gamma$, as defined in [63]. According to Kveder et al. [60], the concentration of recombination active centers $N_{M}$, which is responsible for a certain EBIC contrast of a single dislocation, is defined to be the concentration of deep acceptor levels per unit dislocation length, and $N_{\mathrm{M}}$ might be equal to the concentration of impurity atoms per dislocation length. Assuming a direct proportionality between the EBIC contrast and the recombination activity $\Gamma$ of single dislocations, i.e., of $N_{M}$, which is called linear contrast model [63], then a direct proportionality between the density of Lomer dislocations along a GB and its EBIC contrast can be assumed as well. Indeed, the data shown in the graph of Fig. 8 are in good correlation with the experimental findings of Seifert et al. [48], and the corresponding theory given in [45]. In [48], the correlation between the EBIC contrast and the overall dislocation density was already shown. Now our measurements reveal that only Lomer dislocations contribute to the EBIC contrast of the SAGBs. Furthermore, the share of Lomer dislocation and pd is different in the respective parts of the SAGB, and is dependent on the angle of the SAGB to the lattice planes. Our results should encourage the community to name the typical areas of high recombination in mc-Si solar cells, as shown in Fig. 1(a) (which have been named often just "defects," "GB/defect/dislocation clusters," "recombination active GBs/dislocations," or "type-A defect" [65] in the past) much more precisely now as "Lomer dislocations at SAGBs." In fact, our results point also to the fact that the dominant recombination in mc-Si solar cells happens at inner-grain or subgrain structures, as it was described before by 
other authors as well, for instance [5], [9], [44], and [53]. However, our results show that these subgrain structures are only active if they contain a high density of Lomer dislocations. The role of CSL GBs regarding recombination in mc-Si solar cells seems to be only a minor one. Furthermore, our results revealed that the overall dislocation density is not a good measure for the strength of the recombination, rather the type of the dislocations must be taken into account as well, whereas in previous discussions of the recombination activity of GBs in mc-Si for solar cells often only the $\Sigma$-type of a GB is considered for describing the type of a GB (see, e.g., [44], [50], and [51]).

Although Lomer dislocations are identified as one of the main sources of recombination in mc-Si solar cells, it is still unclear if they contain impurity atoms, e.g., iron, driving the recombination activity $\Gamma$. Typically, Lomer dislocations are able to carry impurity atoms in their core [63]. Maybe the reported immobile nature of the Lomer dislocations contributes to an increased concentration of impurity atoms in their core. However, in the course of this paper, we could not detect any iron, other impurity elements, or precipitates either at the SAGBs or at the Lomer dislocations. This might be due to the fact that the impurity concentration is very low, and only some impurity atoms per Lomer dislocation are enough to yield a high recombination activity. Kveder et al. [63] determined the concentration of impurity atoms per dislocation to be in the order of magnitude from $10 \mu \mathrm{m}^{-1}$ after gettering of impurities (e.g., due to the phosphorus diffusion getter process during the solar cell process) to about $1000 \mu \mathrm{m}^{-1}$ before a getter step. In the latter case, at such high concentrations, it was assumed that one has to deal rather with nanoscaled precipitates than single impurity atoms. However, we could not detect any nanoscaled precipitates, which is due to the fact that our sample saw the whole solar cell process including getter steps. We, thus, assume a very low concentration of impurity atoms of 10 per $\mu \mathrm{m}$. From Figs. 6 and 7, we can estimate the diameter $d$ of a single Lomer dislocation core to be about 0.5 to $1 \mathrm{~nm}$, the FIB lamella has a thickness $h$ of about 50 to $100 \mathrm{~nm}$ (into the paper plane). Hence, following the numbers after gettering taken from [63], we can estimate to have statistically only one impurity atom per dislocation regarding the investigated cylindrical volume of about $\left(\pi / 4 d^{2} h\right)=$ $100 \mathrm{~nm}^{3}$ in the FIB lamellae. Such small numbers of impurity atoms are not detectable by the methods used here.

\section{SUMMARY AND OUTLOOK}

By a combination of DLIT and EBIC solar cell characterization methods, precise target preparation by FIB and electron microscopy investigations, a direct correlation between the dark current losses due to increased diffusion current $J_{01}$ in mc-Si solar cells and the density of Lomer dislocations at SAGBs is revealed. Areas of increased $J_{01}$ in mc-Si solar cells show local efficiency losses of several percent absolute, which is detrimental to the solar cells overall maximum performance. At these SAGBs Lomer dislocations, pd and SFs have been identified by LAADF-STEM investigations. It turns out that only at Lomer dislocations increased EBIC contrasts (i.e., increased recombination activity) are present, which is responsible for the increased dark current losses in solar cells. Furthermore, a direct correlation between the density of Lomer dislocations and EBIC contrast was shown. The density of Lomer dislocation is found to be dependent on the angle between the SAGB and the lattice planes of the surrounding grain. We were not able to detect impurity atoms at the Lomer dislocations in our samples. However, in future, it might be possible to detect such small concentrations of impurity atoms by atom probe tomography [66]. So far, we have only detected Lomer dislocations as being the active parts of the SAGBs at RT.

\section{ACKNOWLEDGMENT}

The authors would like to thank H. Deniz (MPI Halle) for supporting TEM investigations. J. Bauer would like to thank Prof. P. Fratzl (MPI of Colloids and Interfaces, Potsdam, Germany).

\section{REFERENCES}

[1] M. A. Green et al., "Solar cell efficiency tables (version 42)," Prog. Photovoltaic Res. Appl., vol. 21, pp. 827-837, 2013.

[2] J. F. Nijs, J. Szlufcik, J. Poortmans, S. Sivoththaman, and R. P. Mertens, "Advanced manufacturing concepts for crystalline silicon solar cells," IEEE Trans. Electron Devices, vol. 46, no. 10, pp. 1948-1969, Oct. 1999.

[3] S. R. Wenham and M. A. Green, "Silicon solar cells," Prog. Photovoltaics Res. Appl., vol. 4, pp. 3-33, 1996.

[4] G. Hahn and S. Joos, "State-of-the-art industrial crystalline silicon solar cells," in Semiconductors and Semimetals (ser. Advances in Photovoltaics Part 3), G. P. Willeke and E. R. Weber, Eds. 1st ed. San Diego, CA, USA: Academic, 2014, vol. 90, pp. 1-62.

[5] M. Rinio et al., "Recombination in ingot cast silicon solar cells," Phys. status solidi (a), vol. 208, pp. 760-768, 2011.

[6] E. Sirtl, "Future trends in solar silicon production," Sol. Cells, vol. 10, pp. 101-108, 1983.

[7] K. Fujiwara et al., "Growth of structure-controlled polycrystalline silicon ingots for solar cells by casting," Acta Mater, vol. 54, pp. 3191-3197, 2006.

[8] C. W. Lan et al., "Grain control in directional solidification of photovoltaic silicon,” J. Cryst. Growth, vol. 360, pp. 68-75, 2012.

[9] G. Stokkan, Y. Hu, Ø. Mjøs, and M. Juel, "Study of evolution of dislocation clusters in high performance multicrystalline silicon," Sol. Energy Mater. Sol. Cells, vol. 130, pp. 679-685, 2014.

[10] J. Yi, E. R. Weber, C. W. Lan, S. Bremmer, and D. H. Kim, "Photoenergy: Progress in Si-related solar cells for a low cost and high efficiency," Int. J. Photoenergy, vol. 2012, p. 1, 2012.

[11] E. R. Weber, "The future of PV based on next-generation Fabs," presented at the Opening Plenary 6th World Conf. Photovoltaic Energy Conversion, Kyoto, Japan, 2014.

[12] A. Cuevas, "Multicrystalline silicon: The photovoltaic material by excellence," Mater. Forum, vol. 27, pp. 1-8, 2004.

[13] A. A. Istratov et al. " "Metal content of multicrystalline silicon for solar cells and its impact on minority carrier diffusion length," J. Appl. Phys., vol. 94, pp. 6552-6559, 2003.

[14] T. Buonassisi et al., "Transition metals in photovoltaic-grade ingot-cast multicrystalline silicon: Assessing the role of impurities in silicon nitride crucible lining material," J. Cryst. Growth, vol. 287, pp. 402-407, 2006.

[15] A.-K. Søiland et al., "Carbon and nitrogen contents and inclusion formation during crystallization of multi-crystalline silicon," in Proc. 19th Eur. Photovoltaic Sol. Energy Conf., Paris, France, 2004, pp. 911-914.

[16] A. A. Istratov, H. Hieslmair, and E. R. Weber, "Iron contamination in silicon technology," Appl. Phys. A, vol. 70, pp. 489-534, 2000.

[17] G. Coletti et al., "Effect of iron in silicon feedstock on $p$ - and $n$-type multicrystalline silicon solar cells," J. Appl. Phys., vol. 104, art. no. 104913, pp. 104913-1-104913-11, 2008.

[18] G. Coletti et al., "Impact of metal contamination in silicon solar cells," Adv. Funct. Mater, vol. 21, pp. 879-890, 2011.

[19] J. Chen, D. Yang, Z. Xi, and T. Sekiguchi, "Recombination activity of $\Sigma 3$ boundaries in boron-doped multicrystalline silicon: Influence of iron contamination," J. Appl. Phys., vol. 97, art. no. 033701, pp. 033701-1033701-5, 2005. 
[20] W. Shockley and W. T. Read, "Statistics of the recombinations of holes and electrons," Phys. Rev., vol. 87, pp. 835-842, 1952.

[21] G. Zoth and W. Bergholz, "A fast, preparation-free method to detect iron in silicon,” J. Appl. Phys., vol. 67, pp. 6764-6771, 1990.

[22] C. B. Collins and R. O. Carlson, "Properties of silicon doped with iron or copper," Phys. Rev., vol. 108, pp. 1409-1414, 1957.

[23] K. Graff and H. Pieper, "The properties of iron in silicon," J. Electrochem. Soc., vol. 128, pp. 669-674, 1981.

[24] H. Lemke, "Dotierungseigenschaften von eisen in silizium," Phys. status solidi (a), vol. 64, pp. 215-224, 1981.

[25] S. D. Brotherton, P. Bradley, and A. Gill, "Iron and the iron-boron complex in silicon," J. Appl. Phys., vol. 57, pp. 1941-1943, 1985.

[26] S. Rein and S. W. Glunz, "Electronic properties of interstitial iron and iron-boron pairs determined by means of advanced lifetime spectroscopy," J. Appl. Phys., vol. 98, pp. 113711-1-113711-12, 2005.

[27] T. Buonassisi et al., "Synchrotron-based investigations of the nature and impact of iron contamination in multicrystalline silicon solar cells," J. Appl. Phys., vol. 97, art. no. 074901, pp. 074901-1-074901-11, 2005.

[28] A. Hähnel et al., "Electron microscope verification of prebreakdowninducing $\alpha$-FeSi2 needles in multicrystalline silicon solar cells," J. Appl. Phys., vol. 113, art. no. 044505, pp. 044505-1-044505-10, 2013.

[29] M. C. Schubert et al., "Analysis of performance limiting material properties of multicrystalline silicon," Sol. Energy Mater. Sol. Cells, vol. 94, pp. 1451-1456, 2010.

[30] J. L. Benton et al., "Iron gettering mechanisms in silicon," J. Appl. Phys., vol. 80, pp. 3275-3284, 1996.

[31] P. S. Plekhanov, R. Gafiteanu, U. M. Gösele, and T. Y. Tan, "Modeling of gettering of precipitated impurities from $\mathrm{Si}$ for carrier lifetime improvement in solar cell applications," J. Appl. Phys., vol. 86, pp. 2453-2458, 1999.

[32] I. Périchaud, "Gettering of impurities in solar silicon," Sol. Energy Mater. Sol. Cells, vol. 72, pp. 315-326, 2002.

[33] B. Geyer, G. Schwichtenberg, and A. Müller, "Increased wafer yield in silicon ingots by the applications of high purity silicon nitride-coating and high-purity crucibles," in Proc. 31st IEEE Photovoltaic Spec. Conf. Exhib., Lake Buena Vista, FL, USA, 2005, pp. 1059-1061.

[34] R. Kvande, L. Arnberg, C. Martin, "Influence of crucible and coating quality on the properties of multicrystalline silicon for solar cells," J. Crystal Growth, vol. 311, pp. 765-768, 2009.

[35] M. C. Schubert et al., "Impact of impurities from crucible and coating on mc-silicon quality-the example of iron and cobalt," IEEE J. Photovoltaics, vol. 3, no. 4, pp. 1250-1258, Oct. 2013.

[36] W. Kwapil et al., "Influence of crucible and coating on the contamination of directionally solidified silicon: First results of the German research network 'SolarWins,"' in Proc. 27th Eur. Photovoltaic Sol. Energy Conf., Frankfurt, Germany, 2012, pp. 627-635.

[37] J. Schön, H. Habenicht, M. C. Schubert, and W. Warta, "Understanding the distribution of iron in multicrystalline silicon after emitter formation, theoretical model and experiments," J. Appl. Phys., vol. 109, art. no. 063717, pp. 063717-1-063717-8, 2011.

[38] D. P. Fenning et al., "Precipitated iron: A limit on gettering efficacy in multicrystalline silicon," J. Appl. Phys., vol. 113, art. no. 044521, pp. 044521-1-044521-12, 2013

[39] J. Schön et al., "Analyses of the evolution of iron-silicde precipitates in multicrystalline silicon during solar cell processing," IEEE J. Photovoltaics, vol. 3, no. 1, pp. 131-137, Jan. 2013.

[40] W. Kwapil, J. Schön, F. Schindler, W. Warta, and M. C. Schubert, "Impact of iron precipitates on carrier lifetime in as-grown and phosphorous gettered multicrystalline silicon wafers in model and experiment," IEEE J. Photovoltaics, vol. 4, no. 3, pp. 791-798, May 2014.

[41] F. Schindler et al., "Solar cell efficiency losses due to impurities from the crucible in multicrystalline silicon," IEEE J. Photovoltaics, vol. 4, no. 1, pp. 122-129, Jan. 2014.

[42] T. S. Fell, P. R. Wilshaw, and M. D. de Coteau, "EBIC investigations of dislocations and their interactions with impurities in silicon," Phys. status solidi (a), vol. 138, pp. 695-704, 1993

[43] M. Kittler, W. Seifert, and V. Higgs, "Recombination activity of misfit dislocations in silicon," Phys. status solidi (a), vol. 137, pp. 327-335, 1993.

[44] J. Chen and T. Sekiguchi, "Carrier recombination activity and structural properties of small-angle grain boundaries in multicrystalline silicon," Jpn. J. Appl. Phys., vol. 46, pp. 6489-6497, 2007.
[45] C. Donolato, "The relation between EBIC contrast and recombination velocity of a grain boundary," Mater. Sci. Eng., vol. B24, pp. 61-63, 1994.

[46] S. Rißland and O. Breitenstein, "High resolution saturation current density imaging at grain boundaries by lock-in thermography," Sol. Energy Mater. Sol. Cells, vol. 104, pp. 121-124, 2012.

[47] L. Pasemann, H. Blumtritt, and R. Gleichmann, "Interpretation of EBIC contrast of dislocations in silicon," Phys. status solidi (a), vol. 70, pp. 197-209, 1982.

[48] W. Seifert, G. Morgenstern, and M. Kittler, "Influence of dislocation density on recombination at grain boundaries in multicrystalline silicon," Semicond. Sci. Technol., vol. 8, pp. 1687-1691, 1993.

[49] M. Seibt et al., "Gettering in silicon photovoltaics: Current state and future perspectives," Phys. status solidi (a), vol. 203, pp. 696-713, 2006.

[50] J. Chen et al., "Electron-beam-induced current study of grain boundaries in multicrystalline silicon," J. Appl. Phys., vol. 96, pp. 5490-5495, 2004.

[51] Z.-J. Wang, S. Tsurekawa, K. Ikeda, T. Sekiguchi, and T. Watanabe, "Relationship between electrical activity and grain boundary structural configuration in polycrystalline silicon," Interface Sci., vol. 7, pp. 197-205, 1999.

[52] Y. Miyamura, H. Harada, S. Ito, J. Chen, and T. Sekiguchi, "Structural study of small angle grain boundaries in multicrystalline Si," Mater. Sci. Forum, vol. 725, pp. 157-160, 2012.

[53] D. Oriwol et al., "Small-angle subgrain boundaries emanating from dislocation pile-ups in multicrystalline silicon studied with synchrotron whitebeam X-ray topography," Acta Materialia, vol. 61, pp. 6903-6910, 2013.

[54] D. Oriwol, "Die Versetzungsstruktur von multikristallinem Silicium aus der industriellen VGF-Blockkristallisation," Ph.D. dissertation, Brandenburg Univ. Technology Technol., Cottbus-Senftenberg, Germany, 2014.

[55] O. Breitenstein, "Local efficiency analysis of solar cells based on lockin thermography," Sol. Energy Mater. Sol. Cells, vol. 107, pp. 381-389, 2012.

[56] O. Breitenstein, "Nondestructive local analysis of current-voltage characteristics of solar cells by lock-in thermography," Sol. Energy Mater. Sol. Cells, vol. 95, pp. 2933-2936, 2011.

[57] (2015, Jul.). Search for "Local IV 2" [Online]. Available: http://www.maxplanck-innovation.de

[58] J. Bauer et al., "Hot spots in multicrystalline silicon solar cells: Avalanche breakdown due to etch pits," Phys. Status Solidi RRL, vol. 3, pp. 40-42, 2009

[59] J. Bauer et al., "Do Lomer dislocations spoil high performance of mc-Si solar cells?" Energy Procedia, vol. 77, pp. 565-571, 2015.

[60] A. Bourret, J. Desseaux, and A. Renault, "Core structure of the Lomer dislocation in germanium and silicon," Philosoph. Mag. A, vol. 45, pp. 1-20, 1982.

[61] A. H. Cottrell, "The formation of immobile dislocations during slip," Philosoph. Mag., vol. 43, pp. 645-647, 1952.

[62] T. Sekiguchi and K. Sumino, "Cathodoluminescence study on dislocations in silicon,” J. Appl. Phys., vol. 79, pp. 3253-3260, 1996.

[63] V. Kveder, M. Kittler, and W. Schröter, "Recombination activity of contaminated dislocations in silicon: A model describing electron-beaminduced current contrast behavior," Phys. Rev. B, vol. 63, art. no. 115208, pp. 115208-1-115208-11, 2001.

[64] C. Donolato, "Modelling the effect of dislocations on the minority carrier diffusion length of a semiconductor," J. Appl. Phys., vol. 84, pp. 26562664, 1998.

[65] D. Lausch et al., "Classification of recombination-active defects in multicrystalline solar cells made from upgraded metallurgical grade (UMG) silicon," Solid State Phenomena, vols. 178/179, pp. 88-93, 2011.

[66] A. Stoffers et al., "Grain boundary characterization in multicrystalline silicon using joint EBSD, EBIC, and atom probe tomography," in Proc. 40th IEEE Photovoltaic Spec. Conf. Exhib., Denver, CO, USA, 2014, pp. 0042-0046. 